\title{
Fast resolution and good outcomes of isolated traumatic intraventricular haemorrhage: a case report
}

\author{
Mohamed M. Ali ${ }^{1}$, Ali H. Elbasuny ${ }^{1}$, Mostafa Meshref ${ }^{2}$, Odai Hamed Al-ma'aitah ${ }^{3}$, Eshak I. Bahbah ${ }^{4}$ \\ ${ }^{1}$ Neurology Department, Al-Azhar University Hospital, Damietta, Egypt, ${ }^{2} \mathrm{Al}-$ Azhar University, Cairo, Egypt, ${ }^{3}$ School of Nursing and \\ Midwifery, Faculty of Health, University of Technology Sydney, Ultimo, NSW, Australia, ${ }^{4}$ Faculty of Medicine, Al-Azhar University, \\ Damietta, Egypt
}

\begin{abstract}
The location and the size of intracranial haemorrhage play an important role in determining the time for the hematoma resolution. Without surgical intervention, it takes at least a few weeks for the whole hematoma to resolve spontaneously. Despite that the rapid and spontaneous resolution of intracranial haemorrhage is well described in the literature, it is considered rare. Moreover, we could identify only one case of rapid spontaneous resolution of isolated traumatic intraventricular haemorrhage (ITIVH) in the literature. Herein, we report a rare case of ITIVH that disappeared rapidly without surgical intervention. Our case supported the hypothesis that the cerebrospinal fluid (CSF) and its circulation are major players in the rapid resolution of ITIVH.
\end{abstract}

Key words: intraventricular haemorrhage, intracranial haemorrhage, haemorrhagic stroke, cerebrospinal fluid, case report.

\section{Introduction}

Isolated traumatic intraventricular haemorrhage (ITIVH) term is used when intraventricular haemorrhage (IVH) is restricted to the ventricular system and caused by head trauma. It is an extremely rare entity, representing less than $5 \%$ of all cases of isolated $\mathrm{IVH}$ [13]. It is more common in the paediatric population than in adults. Studies conducted to report its incidence concluded different incidence rates ranging from $0.4 \%$ to $4.0 \%$ in all traumatic head patients $[7,8,10]$. Atzema et al. conducted their prospective multicentre study over 8374 patients with head trauma, and they found that the prevalence of isolated traumatic intraventricular haemorrhage was $1.4 \%$ [1]. They reported an overall poor outcome in $70 \%$ of patients. Poor outcomes were associated with a low Glasgow coma scale (GCS; especially less than 8), including the presence of haemorrhage inside the third or fourth ventricles, and the presence of other major injuries in the brain. On the other hand, patients with true isolated IVH were associated with a better outcome.

True isolated IVH is defined by the presence of only intraventricular haemorrhage on both initial and follow-up computed tomography (CT) scans, except for basal ganglia contusion [1]. Isolated IVH can be caused by severe trauma adequate to cause shearing of subependymal veins present in the fornix, septum pellucidum, or choroid plexus [11,17].

Communicating author:

Eshak I. Bahbah, Faculty of Medicine, Al-Azhar University, Damietta, Egypt, 34511, phone: +201094919098 ,

e-mail: Isaacbahbah@gmail.com 
Other causes of isolated IVH are vascular pathologies, arteriovenous malformation (AVM), aneurysm, cavernous malformation, Moyamoya disease, vasculitis, trauma, surgery, and recirculation of subarachnoid haemorrhage (SAH) tumour. Predisposing factors include arterial hypertension, coagulopathy, anticoagulation, and sympathomimetic abuse [2]. In more than $25 \%$ of cases, the cause cannot be reached, despite meticulous search $[9,15]$.

Isolated traumatic IVH is most commonly restricted to the lateral ventricle; however, it may also affect the third and fourth ventricles in isolation or combined with the lateral ventricle [2]. Despite the fact that it is intraventricular, it rarely causes hydrocephalus $[3,11,13,17]$. Early diagnosis of IVH can be attained by CT scan [11]. Depending on the site of haemorrhage, we can divide haemorrhage attributable to traumatic brain injury into five categories: traumatic intracerebral haemorrhage $(\mathrm{TICH})$, traumatic epidural hematoma (TEDH), and traumatic intraventricular haemorrhage (TIVH), traumatic subarachnoid haemorrhage (TSAH), and traumatic subdural hematoma (TSDH). These five forms can develop in isolation or combined with each other. Despite the fact that the time required for resolving these forms of a haemorrhage depends on the amount of bleeding, in the absence of surgical intervention, it takes weeks or even months for spontaneous resolution [4,11]. Herein, we present a case with isolated traumatic intraventricular haemorrhage with rapid disappearance in a few days and excellent outcome.

\section{Case presentation}

A 25-year-old man was admitted to the neurology department following a road traffic accident. At the time of admission, his GCS was E3 M5 V4, and his pupils were mid-sized, equal, and well reacting to light. There was no focal neurological deficit on examination. He had insignificant medical and surgical histories. On admission day, anti-oedema and phenytoin, as prophylaxis for seizures, were started. Initial CT on admission, one hour after the accident, revealed an isolated intraventricular haemorrhage in the left lateralventricle without hydrocephalus (Fig. 1). CT angiography was performed 2 days later in order to rule out any vascular pathology. There were no identified vascular or tumoral lesions (Fig. 2). The conscious level improved until the patient became fully conscious on the $5^{\text {th }}$ day after admission and the patient had no focal neurological deficits. CT brain performed on the $4^{\text {th }}$ day of admission revealed complete resolution of the intraventricular haemorrhage (Fig. 3). The patient was discharged after six days. Then the patient has been arranged to have elective conventional cerebral angiography, which revealed no vascular abnormalities (Fig. 4).

\section{Discussion}

It is established in the literature that TEDH may undergo rapid spontaneous resolution, which may be related to the associated fracture. This may be not related to the absorption itself but to the hema-

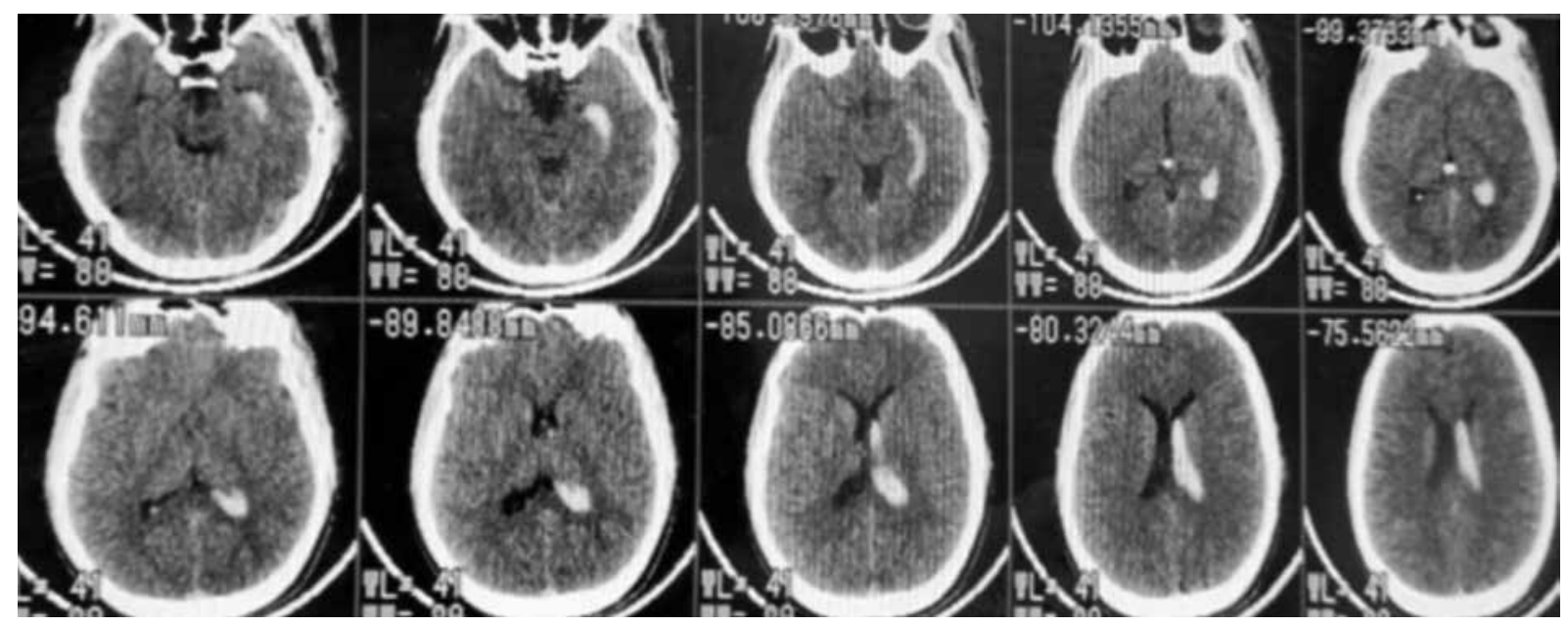

Fig. 1. Non-contrast computed tomography (NCCT) shows isolated intraventricular haemorrhage restricted to the left lateral ventricle. 


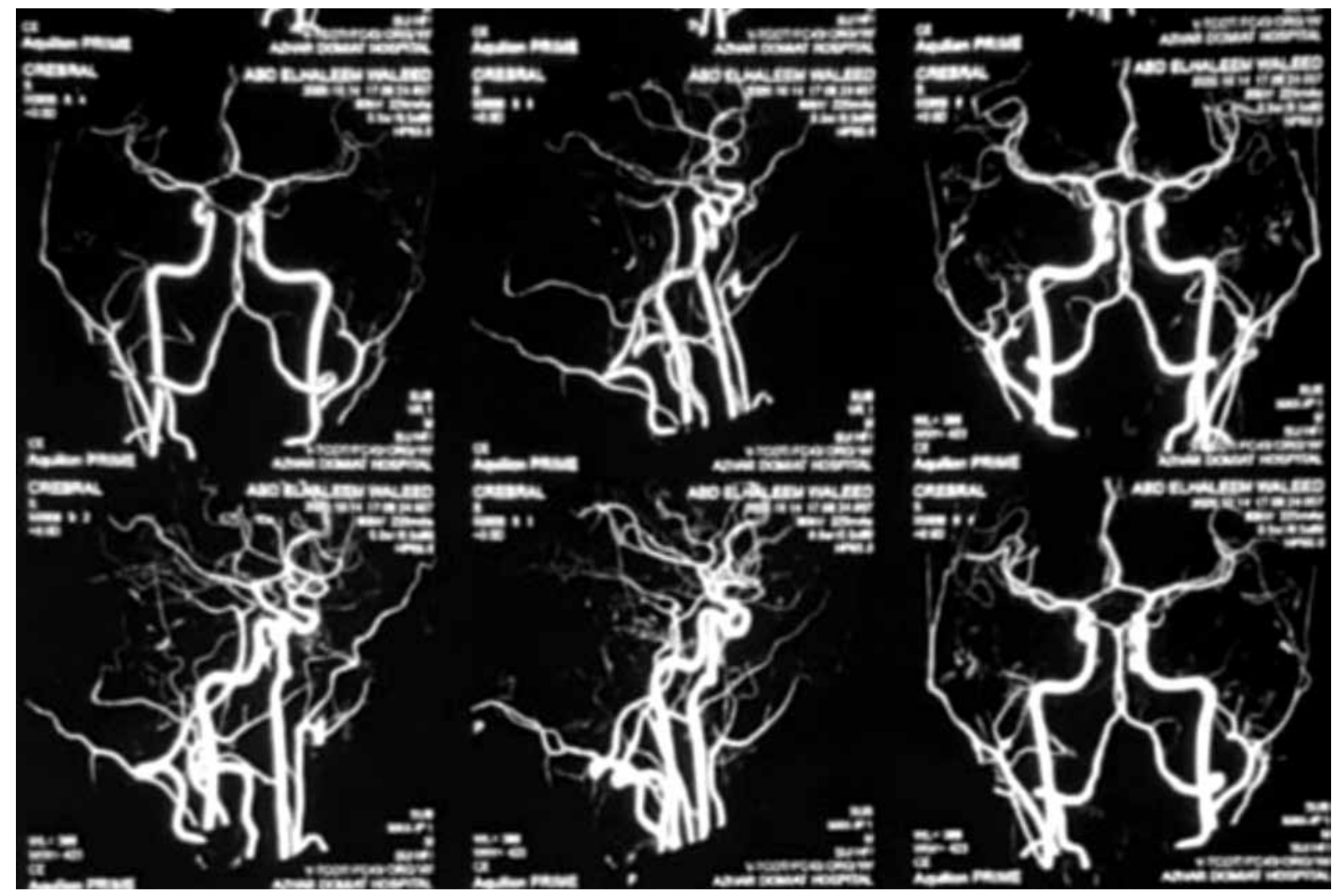

Fig. 2. Computed tomography angiography (CTA) shows no detected abnormalities.

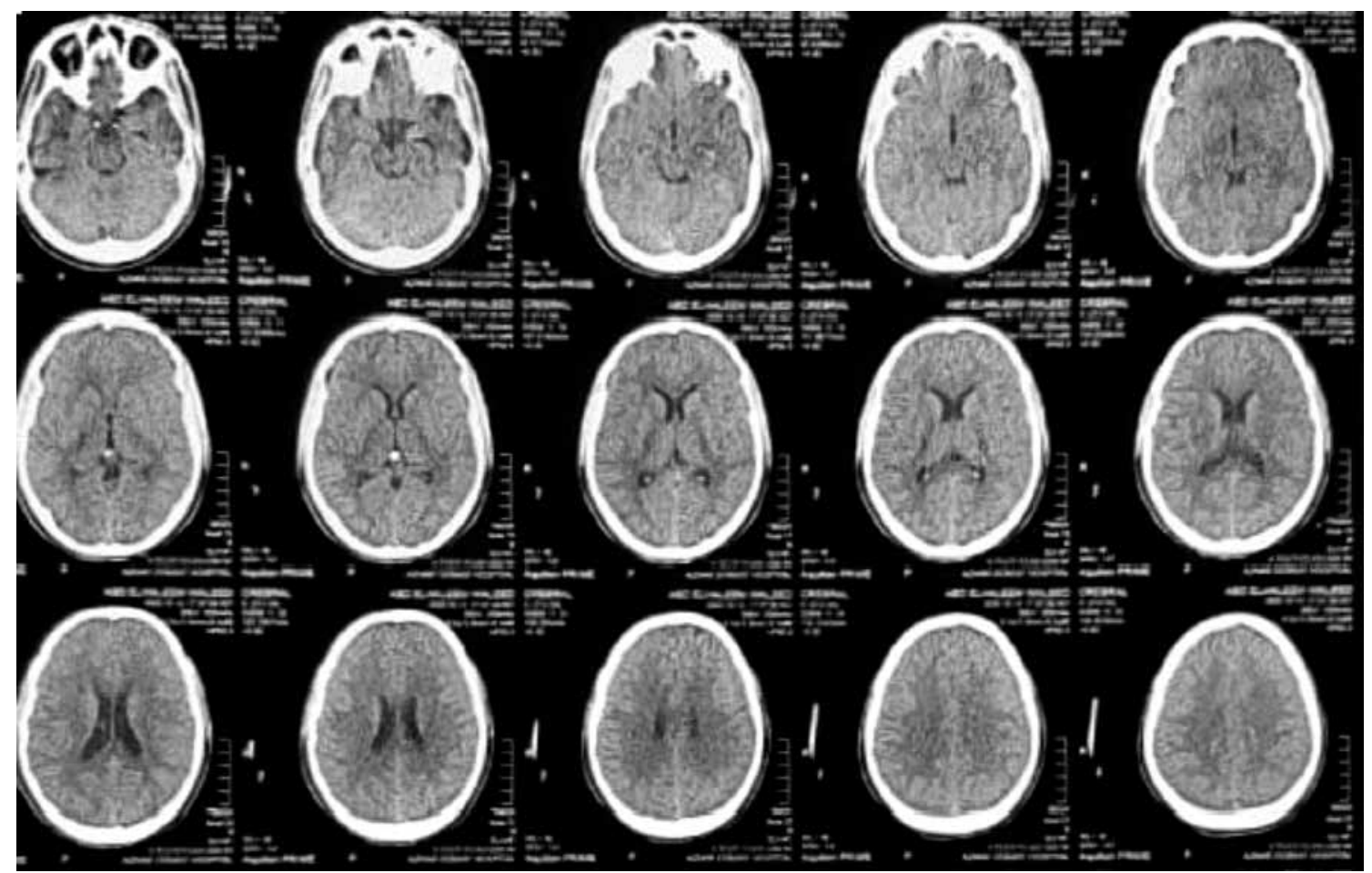

Fig. 3. Non-contrast computed tomography (NCCT) shows resolved intraventricular haemorrhage (IVH) on the $4^{\text {th }}$ day of admission. 

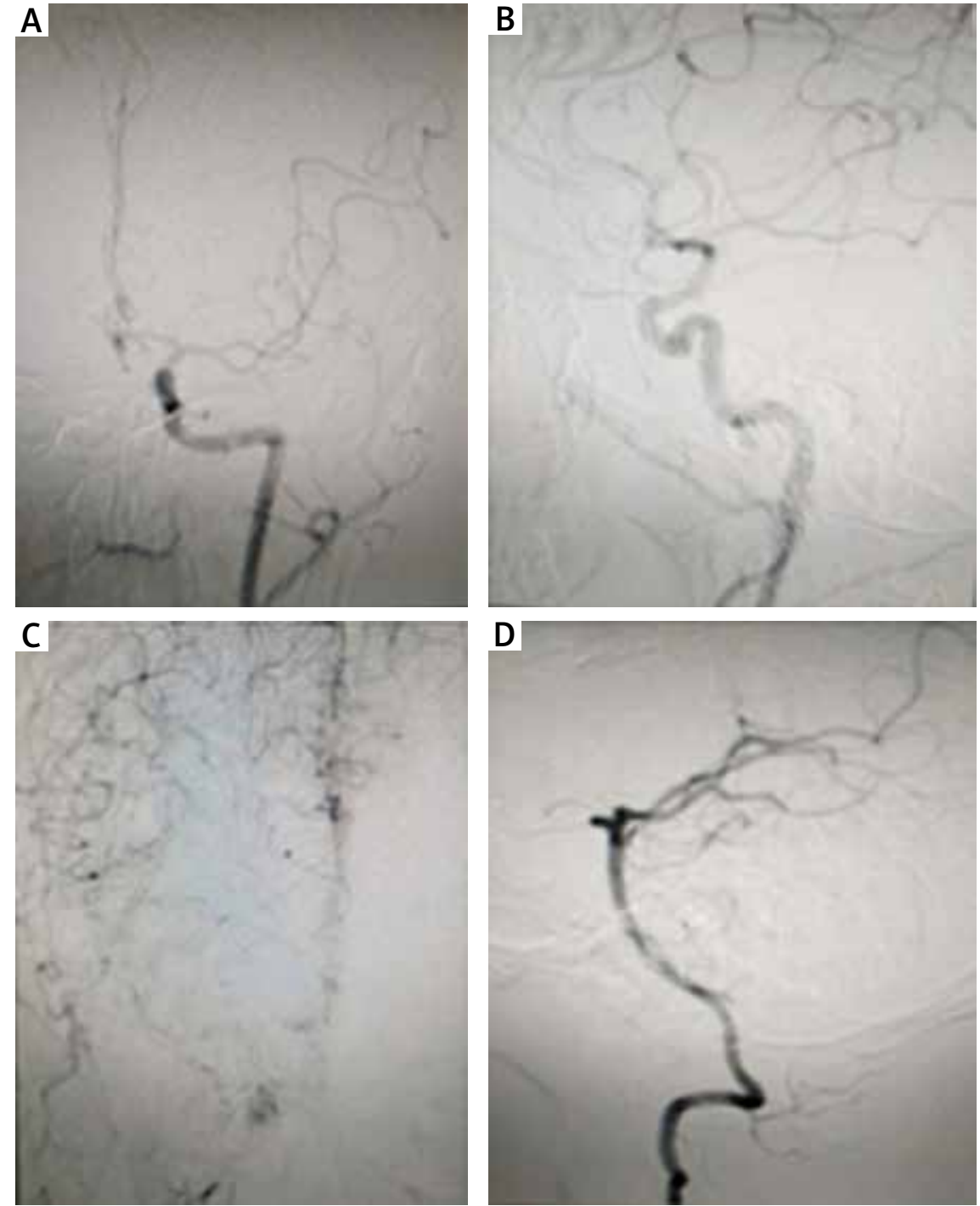

Fig. 4. A) Digital subtraction angiography (DSA) shows normal left internal carotid artery, and terminal branches of anterior cerebral artery and middle cerebral artery. B) DSA shows normal lateral view of the internal carotid artery. C) DSA shows a normal capillary phase of the right internal carotid artery. D) DSA shows normal vertebrobasilar artery and both posterior cerebral arteries.

toma redistribution via the overlying fracture. This may be supported by the observation that patients with TEDH associated with skull fractures have more rapid hematoma resolution than those with TEDH without associated fractures [4]. It is also established, to a lesser extent, that TSDH may undergo spontaneous resolution. This also may be not related to the absorption itself but to the hematoma dilution by leaked cerebrospinal fluid (CSF) through disrupted arachnoid granulation $[11,12]$. This may be supported by the observation of a lower density of hematoma in the case of TSDH $[16,18,19]$. We also identified a case of $\mathrm{TICH}$ with rapid spontaneous resolution [5]. However, we identified only one case report in the literature with rapid resolution of TIVH without surgical intervention. As a conclusion of reviewing many studies discussing the route and mechanisms, hypotheses explaining the spontaneous resolution of traumatic intracranial haemorrhage include extracranial drainage through associated skull fracture and/or disrupted arachnoid and pia membranes. 
A study analysed CT scans of 17 patients with non-traumatic IVH to study the nature of hematoma resolution. They found that the clot resolution rate was not dependent on the initial volume of the hematoma. The rate of drainage was $10.8 \%$ per day. Kinetics for drainage of hematoma was different within the first 48 hours after the drainage. Approximately $29.4 \%$ of patients had an increased haemorrhage by more than $5 \%$ in the first 48 hours. After the initial possible clot expansion, the clot resolves at a constant rate of $10.8 \%$, with a half-life of 5.4 days. There was a complete resolution of IVH at the follow of CT scan on the fourth day of admission in our patient. The absolute rate of the resolution was dependent on the initial volume of haemorrhage, which supports the hypothesis that the system responsible for IVH is the enzyme-substrate system within the CSF [14].

We identified only one case in the literature of isolated traumatic intraventricular haemorrhage with rapid spontaneous and complete resolution reported by Ki Seong Eom [5]. The authors support the hypothesis released by Ki Seong Eom that the CSF has a significant role in rapid dilution and redistribution of hematomas.

In conclusion, we have reported an extremely rare case of ITIVH with spontaneous and rapid disappearance within four days. Despite the ambiguity of that rapid and spontaneous resolution's underlying mechanism, we think that its thrombolytic enzyme system has a critical role in such rapid resolution in the CSF or circulation. More research is needed to explain this rare phenomenon.

\section{Consent} lication.

The patient's consent was obtained for the pub-

\section{Disclosure}

The authors report no conflict of interest.

\section{References}

1. Atzema C, Mower WR, Hoffman JR, Holmes JF, Killian AJ, Wolfson AB. Prevalence and prognosis of traumatic intraventricular hemorrhage in patients with blunt head trauma. J Trauma 2006; 60: 1010-1017.

2. Barnaure I, Liberato AC, Gonzalez RG, Romero JM. Isolated intraventricular haemorrhage in adults. Br J Radiol 2017; 90: 20160779
3. Cordobés F, de la Fuente M, Lobato RD, Roger R, Pérez C, Millán JM, Bárcena A, Lamas E. Intraventricular hemorrhage in severe head injury. J Neurosurg 1983; 58: 217-222.

4. Eom KS, Park JT, Kim TY, Kim JM. Rapid spontaneous redistribution of acute epidural hematoma: Case report and literature review. J Korean Neurosurg Soc 2009; 45: 96-98.

5. Eom KS. A case of rapid spontaneous disappearance of traumatic intraventricular hemorrhage. Korean J Neurotrauma 2020; 16: 273-277.

6. Faheem M, Jaiswal M, Ojha BK, Chandra A, Singh SK, Srivastava C, Saba NU. Disappearance of the hemorrhagic component of the frontal contusion possibly due to redistribution of the hematoma within the subarachnoid space: a case report. Pediatr Neurosurg 2017; 52: 268-270.

7. French BN, Dublin AB. The value of computerized tomography in the management of 1000 consecutive head injuries. Surg Neurol 1977; 7: 171-183.

8. Fujitsu K, Kuwabara T, Muramoto M, Hirata K, Mochimatsu Y. Traumatic intraventricular hemorrhage: report of twenty-six cases and consideration of the pathogenic mechanism. Neurosurgery 1988; 23: 423-430.

9. Giray S, Sen O, Sarica FB, Tufan K, Karatas M, Goksel BK, Yerdelen D, Cekinmez M, Can U. Spontaneous primary intraventricular hemorrhage in adults: clinical data, etiology and outcome. Turk Neurosurg 2009; 19: 338-344.

10. Jayakumar PN, Sastry Kolluri VR, Basavakumar DG, Arya BY, Das BS. Prognosis in traumatic intraventricular haemorrhage. Acta Neurochir (Wien) 1990; 106: 48-51.

11. Karavelis A, Sirmos C. Primary post-traumatic intraventricular hemorrhage. J Neurosurg Sci 1995; 39: 253-256.

12. Kurve A, Mahapatra AK. Spontaneous rapid resolution of traumatic subarachnoid hemorrhage. Indian J Neurotrauma 2005; 2: 47-49.

13. Leroux PD, Haglund MM, Newell DW, Grady MS, Winn HR. Intraventricular hemorrhage in blunt head trauma: An analysis of 43 cases. Neurosurgery 1992; 31: 678-684.

14. Naff NJ, Williams MA, Rigamonti D, Keyl PM, Hanley DF. Blood clot resolution in human cerebrospinal fluid: Evidence of first-order kinetics. Neurosurgery 2001; 49: 614-619.

15. Passero S, Ulivelli M, Reale F. Primary intraventricular haemorrhage in adults. Acta Neurol Scand 2002; 105: 115-119.

16. Shin DW, Choi CY, Lee $\mathrm{CH}$. Spontaneously rapid resolution of acute subdural hemorrhage with severe midline shift. J Korean Neurosurg Soc 2013; 54: 431-433.

17. Tani S, Funahashi K, Hashimoto T, Miyashita A, Sanada S. Traumatic aneurysm of the posterior inferior cerebellar artery (author's transl). No Shinkei Geka 1982; 10: 423-427.

18. Tsui EYK, Fai Ma K, Cheung YK, Chan JHM, Yuen MK. Rapid spontaneous resolution and redistribution of acute subdural hematoma in a patient with chronic alcoholism: a case report. Eur J Radiol 2000; 36: 53-57.

19. Wen L, Liu WG, Ma L, Zhan RY, Li G, Yang XF. Spontaneous rapid resolution of acute subdural hematoma after head trauma: Is it truly rare? Ir J Med Sci 2009; 178: 367-371. 